\title{
Aportes de los estudios comparativos para la comprensión de las políticas y sistemas de salud en países de América Latina
}

\author{
Contribuições dos estudos comparativos para a \\ compreensão das políticas e sistemas de saúde na América Latina
}

\author{
María José Luzuriaga', Lígia Bahia
}

\begin{abstract}
Resumen
Introducción: el objetivo del trabajo es realizar un mapeo en los estudios comparados para identificar las fortalezas y alcances que los mismos tienen en relación a su capacidad para explicar los factores que condicionaron/estimularon los procesos de reforma orientados por la lógica de mercado y de privatización en diversos países y aquellos factores que limitaron o contribuyeron a revertir dicho proceso. Métodos: revisión bibliográfica. Resultados: existe una extensa literatura que ha estudiado los procesos de reforma de salud desde una perspectiva comparada, en los cuales se destaca el peso de las instituciones, los procesos de toma de decisiones, los actores involucrados como las coyunturas críticas. La producción de estudios comparativos tanto de políticas y sistemas de salud de países europeos entre sí y de los mismos con las políticas y sistemas de salud de Canadá y Estados Unidos, ha permitido el desarrollo de debates y reformulaciones teóricas relevantes en términos de hallazgos así como de propuestas teóricas y políticas. En la producción académica de los países de la región no se observa el mismo nivel de desarrollo. Conclusión: avanzar en esta línea de trabajos resulta fundamental para una mejor comprensión de los procesos actuales como para pensar en alternativas de cambio posibles.
\end{abstract}

Palabras clave: estudios comparados; política de salud; sistemas de salud; América Latina.

\section{Resumo}

Introdução: o estudo teve como objetivo realizar um mapeamento nos estudos comparativos para identificar os pontos fortes e o escopo que eles têm em relação à sua capacidade para explicar os fatores que condicionaram/estimularam o processo de reforma guiado pela lógica do mercado e a privatização em vários países como aqueles fatores que contribuíram para limitar ou reverter o processo. Métodos: revisão bibliográfica. Resultados: Existe uma extensa literatura que tem estudado os processos de reforma da saúde em uma perspectiva comparada, que salienta o peso das instituições, processos de tomada de decisão e os grupos interessados, como as conjunturas críticas. A produção de estudos comparativos das políticas e sistemas de saúde em países europeus, e deles com as políticas e sistemas de saúde do Canadá e dos Estados Unidos tem permitido o desenvolvimento de discussões e reformulações teóricas relevantes em termos de resultados e de propostas teóricas e políticas. Na produção acadêmica dos países da região não se observa o mesmo nível de desenvolvimento. Conclusão: o progresso nessa área de trabalho é essencial para uma melhor compreensão dos processos atuais como para pensar sobre possíveis alternativas de mudança. Palavras-chave: estudos comparados; política de saúde; sistemas de saúde; América Latina.

${ }^{1}$ Grupo de Pesquisa e Documentação sobre o Empresariamento na Saúde (GPDES), Instituto de Estudos em Saúde Coletiva, Centro de Ciências da Saúde, Universidade Federal do Rio de Janeiro (UFRJ) - Rio de Janeiro (RJ), Brasil.

Trabajo realizado em el Instituto de Estudos em Saúde Coletiva (IESC), Universidade Federal do Río de Janeiro (UFRJ) - Rio de Janeiro (RJ), Brasil.

Correspondencia: María José Luzuriaga - Avenida Horacio Macedo, sn - Cidade Universitaria - CEP: 21941-598 - Rio de Janeiro (RJ), Brasil -

Email: mariajoseluzuriaga@gmail.com

Apoyo financiero: Beca de doctorado CAPES “Complexo Econômico Industrial da Saúde (CEIS), Inovação e Dinâmica Capitalista: Desafios Estruturais para a Construção do Sistema Universal no Brasil" (CNPq nº 405077/2013-0).

Conflicto de intereses: nada que declarar. 


\section{INTRODUCCIÓN}

El análisis de las dinámicas actuales de los sistemas de salud exige observar la multiplicidad de variables tanto del contexto nacional e internacional así como la compleja trama de estrategias que diversos agentes políticos y sociales internos y externos al campo de la salud han desarrollado en las últimas décadas. Asimismo, analizar las políticas y sistemas de salud requiere definir qué aspectos consideramos más relevantes en cuanto a su capacidad o potencialidad para explicar aquellas contradicciones y vacíos en el conocimiento de modo de poder avanzar en explicaciones que permitan captar de forma más adecuada la realidad estudiada. Sostenemos que una manera de responder a estos desafíos y aproximarnos a una comprensión más rigurosa del tema es posible a partir de una perspectiva comparada.

Para tal objetivo realizamos un mapeo en los estudios comparados - predominantemente del área de políticas de salud y algunos trabajos referentes de las ciencias sociales - direccionado a identificar los objetivos y preguntas formuladas en los mismos para evaluar en qué medida esas formulaciones pueden adecuarse a nuestro tema de estudio y ser capaces de contribuir a nuevas formas de analizar los procesos actuales, en particular las articulaciones público-privado, en los sistemas de salud de nuestros países.

El trabajo se estructura en tres apartados. En primer lugar destacamos los aportes de los estudios comparativos de acuerdo a trabajos referentes de las ciencias sociales. En el apartado siguiente nos ocupamos de los estudios comparados en el campo de la salud. Por un lado, analizamos aquellos que se enfocan en las políticas y sistemas de salud de países europeos y aquellos que comparan los sistemas de países europeos con los sistemas de Canadá y Estados Unidos. En el mismo apartado exponemos aquellos estudios comparativos realizados en América Latina que consideramos más relevantes. Luego se destacan algunos aspectos que deben ser considerados para la aplicación de este tipo de estudios en el campo de la salud. Por último, en el apartado final se realizan las consideraciones finales sobre el uso de estudios comparados para el análisis de las políticas y sistemas de salud de los países de América Latina.

\section{ESTUDIOS COMPARADOS EN LAS CIENCIAS SOCIALES}

Una de las principales referencias en el desarrollo de estudios comparativos en las ciencias sociales la encontramos en Skocpol. En la compilación de estudios que la autora realizó junto a Evans, Rueschemeyer ${ }^{1}$ se encuentra una serie de aportes y aspectos a ser considerados. Entre los más relevantes destacan la posibilidad de relativizar, ponderar y cuestionar explicaciones causales y de ese modo estimular nuevas preguntas e hipótesis de los fenómenos estudiados ${ }^{1}$. También mencionan la capacidad que tienen los mismos para avanzar en un mayor grado de precisión así como la capacidad para buscar explicaciones a las contradicciones y variaciones entre los casos. Como ejemplo, observan en el grupo específico de estudios sobre las capacidades del Estado cómo la perspectiva comparada contribuye a superar la clasificación dicotómica de Estado fuerte o Estado débil a partir de colocar la atención en los contextos internos e internacionales para la comprensión de las políticas estatales ${ }^{2}$. Otro aspecto de gran relevancia en estos estudios se refiere al basamento en la evidencia empírica y la capacidad de poder aproximarnos a aquellos aspectos significativos del problema:

[...] el potencial de los mismos para la realización de estudios empíricos que podrían agudizar nuestro conocimiento de las estructuras del Estado al mismo tiempo que nos puedan permitir tomar una parte significativamente sustantiva de los problemas [...] (p. 361)

Los autores concluyen que es prioritario el desarrollo de estudios comparativos de amplio rango en la medida que "[...] los hallazgos de los mismos le dan a los investigadores la posibilidad de mejorar sus conceptualizaciones y generar nuevas hipótesis sobre la estructura y actividades de los Estados [...]" (p. 363)2.

Otro trabajo que cabe destacar en relación a los aportes de los estudios comparativos es el de Skocpol y Somers ${ }^{3}$ realizado a partir una revisión de estudios histórico-comparativos publicados entre 1960 y 1980. Agrupan a los mismos en tres tipos de acuerdo a los recursos teóricos y metodológicos, y las posibilidades y limitaciones de cada uno de ellos ${ }^{3}$.

Identifican como un primer tipo de estudios a la Demostración Paralela. Este abordaje, busca yuxtaponer los casos históricos con el objetivo de mostrar que una dada hipótesis o teoría puede demostrar su validez cuando "[...] adhiere a una serie

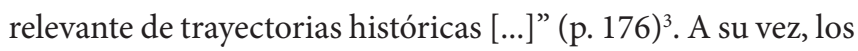
casos son elegidos con el fin de cubrir todas las posibilidades de modo de representar un rango de subtipos o puntos en continuum. Este hecho, según las autoras, además de demostrar repetitivamente la aplicabilidad de la teoría también "[...] enriquece su representación de las formas alternativas posibles de los conflictos sociopolíticos que la teoría está destinada a predecir y explicar [...]" (p. 177) ${ }^{3}$. El segundo caso, denominado Contraste de Contextos, busca traer las características únicas de cada caso para mostrar cómo las mismas son parte del proceso social general bajo estudio. Se considera que los contrastes aumentan la visibilidad y la transparencia del proceso ${ }^{3}$. Así mismo se plantea que el análisis de los contextos permite una mejor comprensión de los mismos y una elaboración más detallada de las inferencias causales. El último tipo de estudio expuesto es el de la Comparación Macro-analítica o Macro-causal, en el cual según Moore

[...] el análisis macro tiende a comparaciones de aspectos relevantes de la historia de dos o más casos [...] ellos tratan de especificar 
configuraciones favorables y desfavorables a resultados particulares que ellos tratan de explicar [...] (p. 182) ${ }^{3}$.

Una última mención que no podemos dejar de considerar en relación a la producción de estudios comparados en las ciencias sociales es la de Esping-Andersen ${ }^{4}$ sobre las tipologías de regímenes de bienestar. Podemos decir que su principal interés se ha centrado en el análisis histórico y comparado de las políticas sociales características de los Estados de Bienestar y cómo las mismas han alcanzado diversos grados de desmercantilización, es decir, "[...] el grado en el cual los individuos y las familias pueden sostener un nivel aceptable de vida independiente de

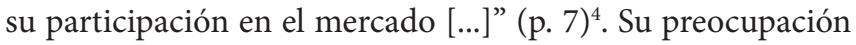
está puesta en evaluar en qué medida las políticas pueden ser tanto emancipadoras de ciudadanía como generar estratificación social. La lectura comparada e histórica le permite al autor identificar los matices y los factores que en los diversos casos han contribuido y aquellos que han limitado al desarrollo de políticas más universales y desmercantilizadas.

Este tipo de propuestas han sido esfuerzos teórico-metodológicos que se inscriben en el campo de las ciencias sociales. Sin embargo, en el campo de la salud podemos afirmar también que ha habido una importante producción de estudios comparativos. En el apartado siguiente expondremos algunos de los abordajes que se han desarrollado en esta línea.

\section{ESTUDIOS COMPARADOS EN EL CAMPO DE}

\section{LA SALUD}

Si intentamos realizar un breve esbozo de los tipos de estudios comparados que se han desarrollado en el campo de la salud podemos identificar en primer lugar aquellos elaborados bajo un abordaje meramente pragmático que predominan en la literatura sobre sistemas de salud desde los años sesenta y setenta. A su vez, también se observa una gran producción de libros (guías) de orientación para comparar sistemas de salud, muchos de los cuales se encuentran inspirados en el trabajo de Talcott Parsons ${ }^{5}$. Otra vertiente prolífica de investigaciones basadas en la comparación entre países se apoya en la cuantificación de la percepción sobre el estatus de salud y el uso de servicios ejemplificada por el estudio clásico sobre las diferencias en la percepción sobre enfermedades y uso de servicios de salud ${ }^{6}$. Por otra parte un estudio de referencia, el trabajo titulado Health Care Systems in the World publicado en 1976 por el médico Milton Roemer ${ }^{7}$ si bien ha sido criticado por basarse en una perspectiva acrítica ${ }^{8}$ fue ampliamente difundido. A partir de los años noventa, las reflexiones sobre los sistemas comparados de salud se volcaron hacia estudios econométricos centrados en el financiamiento y la equidad, dedicados a evidenciar la mayor efectividad de los sistemas de salud europeos universales en contraste con el sistema de EUA? .
Los estudios comparativos y su aplicación al estudio de las políticas de salud

Los trabajos que exponemos a continuación son aquellos que se han enfocado en el análisis comparativo de las políticas de salud y no en los sistemas de salud ya que tomando la revisión sobre estudios comparados realizada por Marmor y Wendt ${ }^{10}$ es una de las distinciones posibles a ser consideradas en los estudios comparados en el campo de la salud. Un primer elemento a destacar en este subgrupo de estudios es que en su mayoría utilizan un abordaje institucionalista. A su vez, es posible identificar algunos temas predominantes. En primer lugar, varios de los mismos se proponen identificar aquellos factores y condiciones que han obstaculizado la implementación de una determinada política o han limitado la realización de sus objetivos. En este grupo podemos destacar el trabajo de Steinmo y Watts ${ }^{11}$, que se preguntan ¿En qué medida las características institucionales de EUA han sido determinantes para que EUA no cuente con un seguro nacional de salud? ${ }^{11}$; o el trabajo de Navarro ${ }^{11}$ que se plantea “¿Por qué algunos países capitalistas desarrollados tienen esquemas de seguro de salud nacional, otros tienen servicios nacionales de salud, y EUA nada? [...]" (p. 85) ${ }^{12}$. Cabe destacar que este autor se ubica en un análisis basado principalmente en una perspectiva marxista. Un segundo grupo centra su análisis en comprender las razones de las divergencias de las configuraciones de los sistemas de salud en contextos socioeconómicos similares y con propuestas de políticas de salud que en algunos casos eran muy similares. En esta línea encontramos los trabajos de Immergut ${ }^{13}$, Wilsford ${ }^{14}$ Hacker ${ }^{15}$ y el de Giaimo ${ }^{16}$ (Cuadro 1). Un tercer grupo analiza el impacto de las reformas de salud y coloca en un primer plano de su análisis a los actores sociales involucrados en el proceso de reforma. En este grupo se pueden destacar los trabajos de Maarse y Paulus ${ }^{17}$ y Hassenteufel et al. ${ }^{18}$ (Ver Cuadro 2).

Identificamos un cuarto grupo de estudios centrados en evaluar y tipificar los sistemas de salud desde la perspectiva del paciente utilizando el concepto de desmercantilización de Esping-Andersen y adaptándolo al análisis de los servicios de salud. Estos estudios elaboran estrategias en relación a una serie de ajustes teórico-metodólogicos que deben considerase para que el concepto de desmercantilización de Esping-Andersen ${ }^{4}$ pueda ser aplicado al campo de las políticas de salud y para evaluar el grado de desmercantilización de las mismas. En esta línea de estudios encontramos principalmente los trabajos de Bambra $^{19}$, Reibling ${ }^{20}$ y Wendt ${ }^{21}$ (Cuadro 3).

Un último trabajo que nos interesa destacar es el de Maarse ${ }^{22}$ que analiza los procesos de privatización de los sistemas de salud. Plantea la complejidad de estudiar los procesos de privatización y la necesidad de desarrollar una conceptualización más específica del tema definiendo las diferentes dimensiones que el proceso envuelve. Propone un marco referencial para analizar los 
procesos de reforma a partir de un análisis comparativo en el cual trabaja con ocho países europeos. El objetivo del trabajo es conocer hasta qué punto se puede observar un proceso de privatización en el cuidado de la salud, es decir, cuáles han sido los cambios en la combinación público-privado. El supuesto que se sostiene es que existen múltiples fronteras en la relación público/privado en la atención de la salud ${ }^{22}$. Una de sus propuestas se centra en la necesidad de incorporar el carácter

Cuadro 1. Estudios comparativos de políticas de salud centrados en Instituciones, dependencias de trayectorias y coyunturas críticas

\begin{tabular}{|c|l|l|l|l|}
\hline Autores & \multicolumn{1}{|c|}{ Immergut } & \multicolumn{1}{c|}{ Giaimo } & \multicolumn{1}{c|}{ Hacker } & \multicolumn{1}{c|}{ Wilsford } \\
\hline Preguntas & $\begin{array}{l}\text { ¿Por qué propuestas de } \\
\text { seguros nacionales de } \\
\text { salud similares resultaron } \\
\text { en configuraciones tan } \\
\text { diferentes? }\end{array}$ & $\begin{array}{l}\text { ¿Cuál fue el impacto de las } \\
\text { reformas de contención de } \\
\text { costos de los 80’y 90' en los } \\
\text { Estado de Bienestar? }\end{array}$ & $\begin{array}{l}\text { ¿Por qué y cuándo ocurren } \\
\text { los cambios? }\end{array}$ & $\begin{array}{l}\text { ¿Por qué y cuándo } \\
\text { suceden los cambios } \\
\text { no incrementales en las } \\
\text { políticas de salud? }\end{array}$ \\
\hline Conceptos & $\begin{array}{l}\text { Intituciones } \\
\text { intereses), } \\
\text { puntos de veto, grupos de } \\
\text { interés, } \\
\text { lobby, trayectorias, } \\
\text { patrones nacionales. }\end{array}$ & $\begin{array}{l}\text { Actores sociales: } \\
\text { sindicatos, corporación } \\
\text { médica, proveedores, } \\
\text { gestores, etc. } \\
\text { Trayectorias } \\
\text { histórico-institucionales, } \\
\text { procesos de toma de } \\
\text { decisión. }\end{array}$ & $\begin{array}{l}\text { Secuencias históricas de } \\
\text { políticas, } \\
\text { ventanas de oportunidad, } \\
\text { dependencia de trayectoria, } \\
\text { coyunturas críticas, } \\
\text { estructura del mercado, } \\
\text { ideas políticas, } \\
\text { visiones de lo público. }\end{array}$ & $\begin{array}{l}\text { Dependencia de trayectoria, } \\
\text { coyunturas críticas, } \\
\text { cambios de trayectorias } \\
\text { graduales, } \\
\text { nuevas trayectorias }\end{array}$ \\
\hline Países & Suiza, Francia y Suecia & Inglaterra, Alemania y EUA & EUA, Canadá e Inglaterra & $\begin{array}{l}\text { Inglaterra, Francia, } \\
\text { Alemania, EUA }\end{array}$ \\
\hline
\end{tabular}

Fuente: Elaboración propia a partir de Immergut ${ }^{13}$, Wilsford ${ }^{14}$, Hacker $^{15}$ y Giaimo $^{16}$

Cuadro 2. Estudios comparativos de políticas de salud con foco en las Instituciones y los Actores sociales

\begin{tabular}{|l|l|l|}
\hline \multicolumn{1}{|c|}{ Autores } & \multicolumn{1}{|c|}{ Maarse y Paulus } & \multicolumn{1}{|c|}{ Hassenteufel et al. } \\
\hline Pregunta/Hipótesis & $\begin{array}{l}\text { ¿Cuál es la influencia de los programas de reformas } \\
\text { sobre la solidaridad en el seguro social de salud? }\end{array}$ & $\begin{array}{l}\text { De la expansión de la competencia interna y la } \\
\text { implementación de mecanismos de cuasi- mercado } \\
\text { en los sistemas de salud no se desprende que "el } \\
\text { mecanismo de funcionamiento es la convergencia } \\
\text { neoliberal". }\end{array}$ \\
\hline Conceptos & $\begin{array}{l}\text { Solidaridad en base a: } \\
\text { - Riesgos } \\
\text { - Ingresos } \\
\text { - Alcance }\end{array}$ & $\begin{array}{l}\text { Actores Programáticos, } \\
\text { ideas, } \\
\text { recursos, } \\
\text { Intereses. }\end{array}$ \\
\hline Países & Bélgica, Alemania, Suiza y Países Bajos & España, Francia, Inglaterra y Alemania \\
\hline
\end{tabular}

Fuente: Elaboración propia a partir de Maarse y Paulus ${ }^{17}$ y Hassenteufel et al. ${ }^{18}$

Cuadro 3. Estudios comparativos de sistemas de salud que utilizan el concepto de desmercantilización

\begin{tabular}{|c|c|c|c|c|}
\hline Autores & Esping-Andersen & Bambra & Reibling & Wendt \\
\hline $\begin{array}{l}\text { Pregunta/ } \\
\text { Propuesta de estudio }\end{array}$ & $\begin{array}{l}\text { ¿Cómo evaluar el grado } \\
\text { de desmercantilización de } \\
\text { una política social? }\end{array}$ & $\begin{array}{l}\text { ¿Cómo adaptar el concepto } \\
\text { de desmercantilización en } \\
\text { los servicios de salud? }\end{array}$ & $\begin{array}{l}\text { Tipología de los sistemas } \\
\text { de salud a partir de la } \\
\text { incorporación del enfoque } \\
\text { del paciente. }\end{array}$ & $\begin{array}{l}\text { Tipología de los sistemas } \\
\text { de salud a partir del acceso } \\
\text { a la atención. }\end{array}$ \\
\hline Conceptos & $\begin{array}{l}\text { Las reglas que gobiernan el } \\
\text { acceso de las personas a los } \\
\text { beneficios tales como: } \\
\text { - las reglas elegibilidad, } \\
\text { - las restricciones sobre los } \\
\text { beneficios, } \\
\text { - el grado de restitución } \\
\text { del salario por los } \\
\text { beneficios de la política } \\
\text { social, } \\
\text { - y el rango de beneficios } \\
\text { provisto por determinada } \\
\text { política. }\end{array}$ & $\begin{array}{l} \\
\text { Índice de } \\
\text { desmercantilización: } \\
\text { - gasto en salud, } \\
\text { - camas de hospitales } \\
\text { privados, } \\
\text { - \% de la población } \\
\text { cubierta por el sistema } \\
\text { público de salud. }\end{array}$ & $\begin{array}{l}\text { Incorporación del enfoque } \\
\text { de Acceso del paciente: } \\
\text { aspectos de la regulación y } \\
\text { los incentivos financieros } \\
\text { que dan forma a la entrada } \\
\text { y la recepción de la } \\
\text { atención. } \\
\text { Indicadores: } \\
\text { control de acceso, } \\
\text { costos compartidos y } \\
\text { suministro. }\end{array}$ & $\begin{array}{l}\text { Acceso a la atención: } \\
\text { - gastos, } \\
\text { - financiamiento, } \\
\text { - prestación, } \\
\text { - acceso a la asistencia. } \\
\text { Acceso a la asistencia } \\
\text { relacionado a: } \\
\text { - niveles de gastos, } \\
\text { - financiamiento público- } \\
\text { privado, } \\
\text { - densidad del servicio de } \\
\text { proveedores. }\end{array}$ \\
\hline
\end{tabular}

Fuente: Elaboración propia a partir de Esping-Andersen ${ }^{4}$, Bambra $^{19}$, Reibling $^{20}$ y Wendt ${ }^{21}$ 
evolutivo del proceso así como de buscar entender los matices que existen en la relación público/privado para comprender el proceso de privatización. Para tal fin propone ver el proceso de privatización "como una secuencia continua de recorridos" (p. 988) ${ }^{22}$. También sostiene que existe una falta de consenso sobre el concepto y por ello resulta necesario analizar el proceso tomando diferentes dimensiones para identificar de forma concreta en qué medida hay elementos privados y públicos, o sea en qué medida podemos definir la existencia y el grado del proceso de privatización así como los escenarios que pueden anticipar tendencias para la privatización ${ }^{22}$.

\section{Estudios comparados de sistemas de salud en América Latina}

En la región encontramos referencias importantes en el desarrollo de estudios comparados que, en su mayoría, han utilizado una perspectiva histórica con énfasis en los cambios institucionales de los sistemas de salud ${ }^{23-31}$. En los mismos se pueden identificar tanto las semejanzas como las diferencias de trayectorias entre los sistemas a partir del análisis de los actores sociales involucrados y los conflictos de interés en juego ${ }^{24,25,27,29}$; los contextos políticos y económicos que han favorecido procesos de reforma ${ }^{25-28,30}$; el rol de los organismos internacionales ${ }^{29,31}$; los procesos de implementación de las reformas y los resultados en términos de igualdad y universalidad de acceso ${ }^{25,26,28,30}$, entre otros. A continuación destacamos aquellos que han desarrollado con mayor especificidad su propuesta teórica y aquellos que han sido considerados como referencias.

Podemos identificar como trabajo pionero en la región el desarrollado por Mesa-Lago ${ }^{23}$, quien inició su trayectoria en esta línea de estudios a comienzos de los 80. Si bien como sostiene Valencia Lomeli ${ }^{32}$, la tradición comparativa de este autor puede también ser colocada en los estudios sobre regímenes de bienestar, en la medida que se centra en los sistemas de seguridad social avanza en el estudio sobre las formas en que el Estado y la sociedad responden a la cuestión de la salud. Elabora una tipología de los sistemas de seguridad social según las trayectorias que han seguido los sistemas en los diversos países y el alcance de los mismos en términos de universalidad de la cobertura, financiamiento, entre otras dimensiones ${ }^{23}$. Resulta interesante el análisis de uno de sus primeros trabajos en torno al rol de los grupos de presión en la conformación de los sistemas de seguridad social a partir de los casos de Argentina, Chile, México, Perú y Uruguay. Elabora un ranking en términos del nivel de inequidades existentes en los diferentes sistemas de seguridad social. La hipótesis principal es la siguiente:

[...] cuanto más poderoso el grupo, más temprano ganaron protección, más elevado es el grado de cobertura, menos deben pagar para financiar el sistema, y mayor es la cantidad como la calidad así como menos estrictas las condiciones para adquirir los beneficios [...] (p. 258) $)^{24}$.
Fleury et al. ${ }^{25}$ realizaron un trabajo cuyo objetivo fue investigar las transformaciones en los modos de financiación y prestación sanitaria resultante de las dos últimas décadas de reformas sociales en Argentina, Brasil y México. El análisis se enfoca en los principales actores involucrados y en los diferentes procesos seguidos durante la implementación. La hipótesis sostenida es que el remodelado de los sistemas de salud a lo largo de 20 años creó amplia libertad de elección entre los proveedores, la coordinación se volvió difícil, y se agravaron las inequidades respecto a la accesibilidad, el uso y la calidad de los servicios en el sistema de salud ${ }^{25}$. Entre las conclusiones destacamos tres cuestiones que consideramos relevantes. En primer lugar, identifican que un factor crucial en los tiempos de los procesos fueron los contextos de crisis económicas y el proceso de democratización así como el proceso de descentralización. Otro aspecto, considerado por las autoras el cambio más importante, y el que afecta la dinámica entera del sector, fue el rol del mercado competitivo en el seguro y en la provisión de la atención de la salud ${ }^{25}$. Por último, postulan que en ninguno de los procesos de reforma se ha colocado en el centro de las mismas la cuestión de la solidaridad a través de los diferentes estratos socioeconómicos en un contexto de sociedades altamente desiguales ${ }^{25}$.

Eliana Labra ${ }^{27}$ por su parte, al igual que Fleury et $a l .{ }^{25}$ centra su análisis en los actores sociales para estudiar las diferentes trayectorias seguidas por los sistemas de salud de Chile y Brasil ${ }^{27}$ pero con mayor énfasis en las instituciones de salud y fundamentalmente en los regímenes políticos. El eje de su análisis son las modernizaciones neoliberales en la década del ochenta bajo la dictadura de Pinochet y las reformas estatizantes emprendidas en Brasil en la misma década. Dos tesis son colocadas por la autora como centrales: "[...] el desarrollo político es la dimensión relevante para explicar los procesos de decisión relativos a las políticas de salud y la configuración diferente de cada sistema [...]" (p. 361 $)^{27}$; y la segunda postula que "[...] el legado histórico de cada construcción institucional es capaz de resistir a los cambios, tanto en la democracia como en la dictadura [...]" (p. 361) ${ }^{27}$.

Un tercer trabajo que comparte con los anteriores la perspectiva histórica y podríamos decir, sociopolítica, es el de Mario Hernández, quien ubica a su trabajo en dicha perspectiva y recupera elementos teóricos del trabajo de Fleury et al. ${ }^{25}$. A partir del estudio de los sistemas de salud de Argentina y Colombia propone un estudio de lo político entendiendo al mismo como un análisis que busca "[...] profundizar en la especificidad de las relaciones políticas que constituyen los procesos por los cuales se construyen las políticas sociales en los Estados [...]" (p. 63) ${ }^{29}$. El eje en lo político supone centrarse en el proceso político y no en las técnicas utilizadas para llevar a cabo las políticas, es decir, que el foco lo coloca en los aparatos y las decisiones políticas ${ }^{29}$. Su hipótesis es que: 
Las diferencias en la organización de los servicios de salud se explican por la configuración particular de las relaciones entre los actores sociopolíticos del campo de la salud de cada país, en relación con la configuración institucional en la que se encuentran, durante períodos relativamente largos [...] (p. 19) ${ }^{29}$.

Desde otra perspectiva, de rechazo a los sistemas universales basados en la oferta pública de cuidados de salud, encontramos el trabajo de Londoño y Frenk ${ }^{33}$ muy citado por los estudios de políticas de salud en la región y que realiza una lectura de los sistemas de salud con el fin de elaborar un modelo "superador" para la región. Aspectos como la dualidad de los sistemas, la cobertura deficiente, la existencia de incentivos inadecuados, el pluralismo creciente, la fragmentación institucional son colocados como los problemas centrales de acceso a los servicios de salud ${ }^{27}$. Proponen un modelo que denominan Pluralismo Estructurado, como modo de superar las fallas en los modelos y la función de modulación para lo cual proponen el desarrollo de organizaciones para la articulación de servicios de salud:

En suma el reto del pluralismo estructurado es incrementar las opciones para los consumidores y los proveedores, y contar al mismo tiempo con reglas de juego explícitas que minimicen los conflictos potenciales entre equidad y eficiencia [...] (p. 17) $)^{33}$

Entre las críticas a este modelo, González Block ${ }^{25}$ destaca la simplificación del análisis de los actores, instituciones y los aspectos normativos en cada caso así como la simplificación o negación de aspectos ideológicos y políticos. Almeida ${ }^{28}$, también realiza una fuerte crítica mostrando que los objetivos que prometía la propuesta elaborada por Lodoño y Frenk no fueron alcanzados donde se implementó y aún así se pretendió usar como modelo para otros países de la región.

Aspectos a ser considerados para el uso de estudios comparados en el estudio de las políticas y sistemas de salud

Marmor y Wendt ${ }^{10}$ realizaron en 2012 una revisión de la producción académica con el fin de identificar los marcos conceptuales que han sido utilizados por los estudios comparativos en políticas y sistemas de salud y en los mismos identificaron tres problemas predominantes: la falta del conocimiento de los contextos locales en que las políticas se implementan; la falta de análisis de los resultados de las políticas; y la falta de atención en los estudios sobre actores políticos involucrados en los procesos políticos ${ }^{10}$.

En relación a la primera dificultad, Klein ${ }^{34}$ llama la atención sobre la existencia de una epidemia de estudios que consiste en importar experiencias e ideas de otros países sin que las mismas hayan sido evaluadas en sus lugares de origen ${ }^{34}$. Y, en ese sentido, propone un análisis profundo del entorno de las políticas y critica todo intento, de organismos internacionales como el Banco Mundial, de proponer un modelo unificado para todos los países ${ }^{34}$.
Otro aspecto que Klein advierte es sobre la no neutralidad del uso de las comparaciones que debe ser tenido en cuenta por los analistas de las políticas y en este punto cita un trabajo de Marmor y Plowden: "Las ideas son elementos en la guerra política cuyo levantamiento es determinado no por su validez intrínseca sino por su ubicación local -en sus modos presentes, circunstancias y estructuras [...]" (p. 1270) ${ }^{34}$.

Por su parte Kieke et al. ${ }^{35}$, plantean la proliferación de estudios comparativos en las décadas recientes y algunos problemas que identifican entre los cuales destacan que "La mayoría de esos estudios, sin embargo, consisten en una colección descriptiva de estudios de casos. En general carecen de un vocabulario común y sufren de pobres definiciones de términos [...]" (p. 76) ${ }^{35}$. Otro problema que observan es que los mismos confunden la comprensión de lo que es la política en los documentos y la política implementada.

Por último, un trabajo de revisión realizado en 2015 que analiza el uso de las comparaciones internacionales, destaca una serie de cuidados que deben ser considerados en el uso de este tipo de metodología:

La tarea de tratar de comparar sistemas de salud internacionalmente continúa siendo difícil. La realización de un ranking puede variar, y de hecho lo hace, significativamente dependiendo el peso y el valor asignado a las métricas específicas o a los temas. Mientras que las tablas de clasificación de los sistemas de atención de la salud continúan siendo producidas [...], expertos, incluyendo el National Quality Board, han cuestionado su utilidad [...]. Aún sin tablas de clasificación, las comparaciones internacionales de desempeño del cuidado de la salud a nivel nacional pueden tener un vacío de significado sin una comprensión detallada del contexto en el que se encuentran los mismos, o sin observar el rango de las medidas y las fuentes de información ${ }^{36}$.

\section{A MODO DE CONCLUSIÓN}

En el recorrido que nos hemos propuesto se ha buscado profundizar el conocimiento en torno a las fortalezas y las limitaciones de los estudios comparados para identificar aquellas propuestas teóricas y metodológicas capaces de captar de una manera más adecuada las dinámicas especificas que han conformado y conforman los procesos de privatización en el campo de la salud así como los aspectos que puedan contribuir a pensar la forma de revertir los mismos.

Cabe destacar que este trabajo es parte de una tesis de doctorado cuyo objetivo es analizar en qué medida es posible un proceso de desprivatización de los sistemas de salud en países de América Latina. Hemos observado la existencia de una amplia producción académica en países europeos y de América del Norte que estudiaron comparativamente tanto los procesos de reforma de salud orientados por el mercado como aquellos procesos que han resistido a procesos privatizantes o han implantado sistemas o seguros nacionales de salud. Es este 
tipo de producción académica la que no hemos encontrado en la misma medida en los países de nuestra región.

Por otra parte, si bien sostenemos la relevancia y la pertinencia que adquieren dichos estudios, consideramos igual de relevante las precauciones que deben tenerse en cuenta sobre los límites de su alcance como transposiciones incorrectas o lecturas simplistas preocupadas en clasificar el desempeño de los mismos que pueden conducir a interpretaciones y resultados inválidos.

Postulamos la necesidad del análisis comparativo como una alternativa con gran potencial para contribuir a una comprensión más rigurosa y completa de los procesos de elaboración e implementación de políticas, como el caso de la privatización de los sistemas de salud. Es a partir de los motivos que hemos mencionado que entendemos la relevancia de poder avanzar en esta línea de trabajos en el campo de las políticas de salud y de este modo fortalecer y consolidar el conocimiento teórico y empírico del tema en esta región. Conocimiento que pretende contribuir al debate público y a la elaboración de políticas públicas para que las mismas puedan intervenir de manera efectiva en el avance de sistemas de salud más justos e igualitarios para toda la sociedad.

\section{REFERENCIAS}

1. Skocpol T. Bringing the State back in: strategies of analysis in current research. In: Evans P, Rueschemeyer D, Skocpol T, editors. Bringing the State back in. Cambridge: Cambridge University Press; 1985. p. 3-37.

2. Evans P, Rueschemeyer D, Skocpol T. On the road toward a more adequate understang of the State. In: Evans P, Rueschemeyer D, Skocpol T, editors. Bringing the State back in. Cambridge: Cambridge University Press; 1985. p. 347-365.

3. Skocpol T, Somers M. The uses of comparative history in macrosocial inquiry. Comp Stud Soc Hist. 1980;22(2):174-97. http://dx.doi.org/10.1017/ S0010417500009282.

4. Esping-Andersen G. As três economias políticas do welfare state. Lua Nova. 1991;24(24):85-116. http://dx.doi.org/10.1590/S0102-64451991000200006.

5. Parsons T. Societies: evolutionary and comparative perspectives. New Jersey: Prentice-Hall; 1966.

6. Bice TW, White KL. Factors related to the use of health services: an international comparative study. Med Care. 1969;7(2):124-33. http://dx.doi. org/10.1097/00005650-196903000-00006. PMid:5798989.

7. Roemer MI. Health care systems in world perspective. Michigan: Health Administration Press, School of Public Health, The University of Michigan and Ann Arbor; 1976.

8. Sidel VW. International comparisons of health services: how? who? why? Policy Stud J. 1980;9(2):300-7. http://dx.doi.org/10.1111/j.1541-0072.1980. tb02207.x.

9. Evans DB, Tandon A, Murray CJL, Lauer JA. Comparative efficiency of national health systems: cross national econometric analysis. BMJ. 2001;323(7308):307-10. http://dx.doi.org/10.1136/bmj.323.7308.307. PMid:11498486.

10. Marmor T, Wendt C. Conceptual framekorks for comparing healthcare politics and policy. Health Policy. 2012;107(1):11-20. http://dx.doi. org/10.1016/j.healthpol.2012.06.003. PMid:22763200.

11. Steinmo S, Watts J. It's the institutions, stupid! Why comprehensive national health insurance always fails in America. J Health Polit Policy Law. 1995;20(2):329-72. http://dx.doi.org/10.1215/03616878-20-2-329. PMid:7636126.

12. Navarro V. Why some countries have National Health Insurance, others have National Health Services, and the U.S has neither? Soc Sci Med. 1989;28(9):887-98. http://dx.doi.org/10.1016/0277-9536(89)90313-4. PMid:2652327.
13. Immergut E. Health politics: interests and institutions in Western Europe. Cambridge: Cambridge University Press; 1992.

14. Wilsford D. Path dependency, or why history makes it difficult but not impossible to reform health care systems in a big way. J Public Policy. 1994;14(03):251-83. http://dx.doi.org/10.1017/S0143814X00007285.

15. Hacker JS. The historical logic of national health insurance: structure and sequence in the development of British Canadian, and U.S. medical policy. Stud Am Polit Dev. 1998;12(01):57-130. http://dx.doi.org/10.1017/ S0898588X98001308.

16. Giaimo S. Who pays for health care reform. In: Pierson P, editor. The new politics of Welfare State. New York: Oxford University Press; 2001. p. 334367. http://dx.doi.org/10.1093/0198297564.003.0012.

17. Maarse H, Paulus A. Has solidarity survived? A comparative analysis of the effect of social health insurance reform in four European countries. J Health Polit Policy Law. 2003;28(4):585-614. http://dx.doi.org/10.1215/0361687828-4-585. PMid:12956517.

18. Hassenteufel P, Smyrl M, Genieys W, Moreno-Fuentes FJ. Programmatic actors and the transformation of European health care states. J Health Polit Policy Law. 2010;35(4):517-38. http://dx.doi.org/10.1215/03616878-2010015. PMid:21057096.

19. Bambra C. Worlds of welfare and the health care discrepancy. Soc Policy Soc. 2010;4(1):31-41. http://dx.doi.org/10.1017/S1474746404002143.

20. Reibling N. Healthcare systems in Europe: towards an incorporation of patient access. J Eur Soc Policy. 2010;20(1):5-18. http://dx.doi. org/10.1177/0958928709352406.

21. Wendt C. Mapping European healthcare systems: a comparative analysis of financing, service provision and access to healthcare. J Eur Soc Policy. 2009;19(5):432-45. http://dx.doi.org/10.1177/0958928709344247.

22. Maarse H. The privatization of health care in Europe: an eight-country analysis. J Health Polit Policy Law. 2006;31(5):981-1014. http://dx.doi. org/10.1215/03616878-2006-014. PMid:17102141.

23. Mesa-Lago C. Efectos de la crisis global sobre la seguridad social de salud y pensiones en América Latina. Santiago de Chile: División de Desarrollo Social, CEPAL; 2009. (Serie Politicas Sociales).

24. Mesa-Lago C. Social Security in Latin America: pressure groups, stratification, and inequality. Pittsburgh: University os Pittsburgh Press; 1978. 
25. Fleury S, Belmartino S, Baris E. Reshaping health care in America Latina a comparative analysis of health care reform in Argentina, Brazil, and México. Otawa: International Development Research Centre; 2000.

26. Fleury S. ¿Universal, dual o plural? Modelos y dilemas de la atención de la salud en América Latina: Chile, Brasil y Colombia. In: Molina CG, Núñez del Arco J. Servicios de salud en América Latina y Asía. Washington: BID/Instituto Interamericano para el Desarrollo Social; 2003. p. 3-37.

27. Labra ME. Políticas e saúde no Chile e no Brasil: contribuições para uma comparação. Cien Saude Colet. 2001;6(2):361-76. http://dx.doi.org/10.1590/ S1413-81232001000200007.

28. Almeida C. Reforma del sector salud y equidad en América Latina y el Caribe: conceptos, agenda, modelos y algunos resultados de implementación. Rev. Gerencia y Politicas de Salud. 2005;4(9):6-60.

29. Hernández Álvarez M. La fragmentación de la salud en Colombia y Argentina: una comparación sociopolítica, 1880-1950. Bogotá: Universidad Nacional de Colombia; 2004.

30. Homedes N, Ugalde A. Privatización de los servicios de salud: las experiencias de Chile y Costa Rica. Gac Sanit. 2002;16(1):54-62. http://dx.doi.org/10.1016/ S0213-9111(02)71633-6. PMid:11841756.
31. Iriart C, Merhy E, Waitzkin H. La atención gerenciada en América Latina: su papel en la reforma de los sistemas de salud. Buenos Aires: Instituto de Estudios y Formación-Central de los Trabajadores Argentinos; 1999.

32. Valenica Lomelí E. Los debates sobre los regímenes de bienestar en América Latina y en el Este de Asia: los casos de México y Corea del Sur. Espiral. 2010;16(47):65-103.

33. Londoño, JL, Frenk J. Pluralismo estructurado: hacia un modelo innovador para la reforma de los sistemas de salud de América Latina. Washington: Oficina del Economista Jefe, Banco Interamericano de Desarrollo; 1997. (Documento de Trabajo, 353).

34. Klein R. Learning from others: shall the last be the first? J Health Polit Policy Law. 1997;22(5):1267-78. PMid:9394248.

35. Kieke GHO, Cheng T, Chinitz D, Crivelli L, Lim M, Maarsef H, et al. Six countries, six health reform models? health care reform in Chile, Israel, Singapore, Switzerland, Taiwan and The Netherlands. JCPA. 2010;12(2):75-113.

36. Berry N. How does the NHS Compare with health systems in other countries? Topic overview. London: The Health Foundation; 2015.

Recibido en: Sept. 16, 2015 Aprobado en: Enero 18, 2016 